Anaesthesist 2016 65:866-871

DOI 10.1007/s00101-016-0226-8

Online publiziert: 5. Oktober 2016

(c) Der/die Autor(en) 2016. Dieser Artikel ist eine Open-Access-Publikation.

CrossMark

\author{
J. Abram ${ }^{1} \cdot \mathrm{J}_{\text {Klocker }}{ }^{2}$ N. Innerhofer-Pompernigg' $\cdot$ M. Mittermayr' \\ M. C. Freund ${ }^{3} \cdot$ N. Gravenstein ${ }^{4} \cdot$ V. Wenzel $^{5}$ \\ ${ }^{1}$ Univ.-Kinik für Anästhesie und Intensivmedizin, Medizinische Universität Innsbruck, Innsbruck, Österreich \\ ${ }^{2}$ Univ.-Klinik für Gefäßchirurgie, Medizinische Universität Innsbruck, Innsbruck, Österreich \\ ${ }^{3}$ Univ.-Klinik für Radiologie, Medizinische Universität Innsbruck, Innsbruck, Österreich \\ ${ }^{4}$ Univ.-Klinik für Anästhesie und Intensivmedizin, University of Florida, Gainesville, USA \\ ${ }^{5}$ Bodensee Medizin Campus, Klinik für Anästhesie, Intensivmedizin, Notfallmedizin und Schmerztherapie, \\ Friedrichshafen, Deutschland
}

\title{
Verletzungen herznaher Gefäße durch zentralvenöse Katheter
}

Angiographie des Aortenbogens und der A. subclavia (• Abb. 2); nach Blockieren der A. subclavia durch einen Okklusions-Ballonkatheter konnten die Katheter zurückgezogen werden und die Perforationsstellen der A. subclavia wurden mit einer beschichteten selbstexpandierenden Metallendoprothese versorgt. Die Kontrollangiographie zeigte ein Sistieren der Blutungsquelle und eine unauffällige Perfusion des Armes.

\section{Fall 2}

Eine 50-jährige Patientin wurde aufgrund einer spontanen Subarachnoidalblutung aus einem A. basilaris Aneurysma in der Notfallaufnahme intubiert. Anschließend wurde ein ZVK in die rechte V. subclavia gelegt, allerdings verliefen Aspirationsversuche aus allen Lumina frustran. Danach kam es bei einem erneuten Punktionsversuch zu einer arteriellen Punktion; die Punktionsstelle wurde dann für drei Minuten komprimiert. Bei einem dritten Ver- such gelang es, den ZVK in die rechte $\mathrm{V}$. subclavia regelrecht $\mathrm{zu}$ platzieren und von den fünf Lumina ließen sich vier problemlos aspirieren. Das Thoraxröntgen zeigte anschließend eine ausgeprägte Verschattung des rechten Hemithorax; der Hämatothorax wurde dann durch eine Bülau-Drainage entlastet. Der ZVK wurde anschließend entfernt und die Perforationsstellen der A. und V. subclavia wurden mit einem beschichteten, selbstexpandierbaren Stent behandelt (•Abb. 3). Vor Einbringen des Mikrokatheters in den zerebralen Aneurysmasack kam es dann jedoch $\mathrm{zu}$ einer massiven Rezidivblutung und $\mathrm{zu}$ einer hochgradigen intrakraniellen Perfusionseinschränkung. Durch die Anlage einer externen Ventrikeldrainage und durch eine maximale ICP-senkende Therapie konnte anschließend eine intrakranielle Perfusion erreicht werden. Die während der Nachblutung eingetretene Hypoperfusion führte jedoch $\mathrm{zu}$ einer ausgeprägten zerebralen Pathologie und zum Eintreten einer therapierefraktären, rückziehen konnte die Schleuse nach intraarteriell manövriert werden; eine vollständige Entfernung der Schleuse war aufgrund der hohen Blutungsgefahr nicht möglich. Vielmehr wurde zusätzlich ein 3-lumiger ZVK in die A. subclavia eingelegt, um die Punktionsstellen an der Gefäßvorder- und -hinterwand zu tamponieren. Die Lebertransplantation konnte nach entsprechender zeitlicher Verzögerung erfolgreich durchgeführt werden. Am nächsten Tag folgte eine

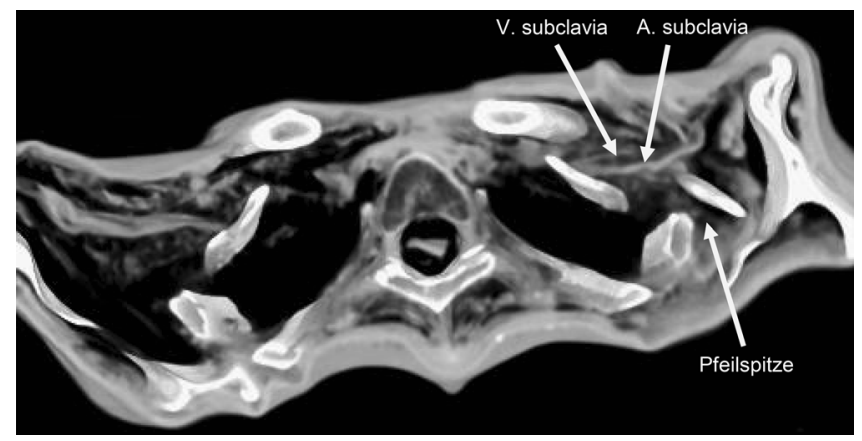

Abb. 14 "Ötzis" Verletzung der A. subclavia durch eine Pfeilspitze (thin-slab MIP) (mit freundl. Genehmigung von Dr. Patrizia Pernter und der Abteilung für Radiologie des Zentralkrankenhauses Bozen) 


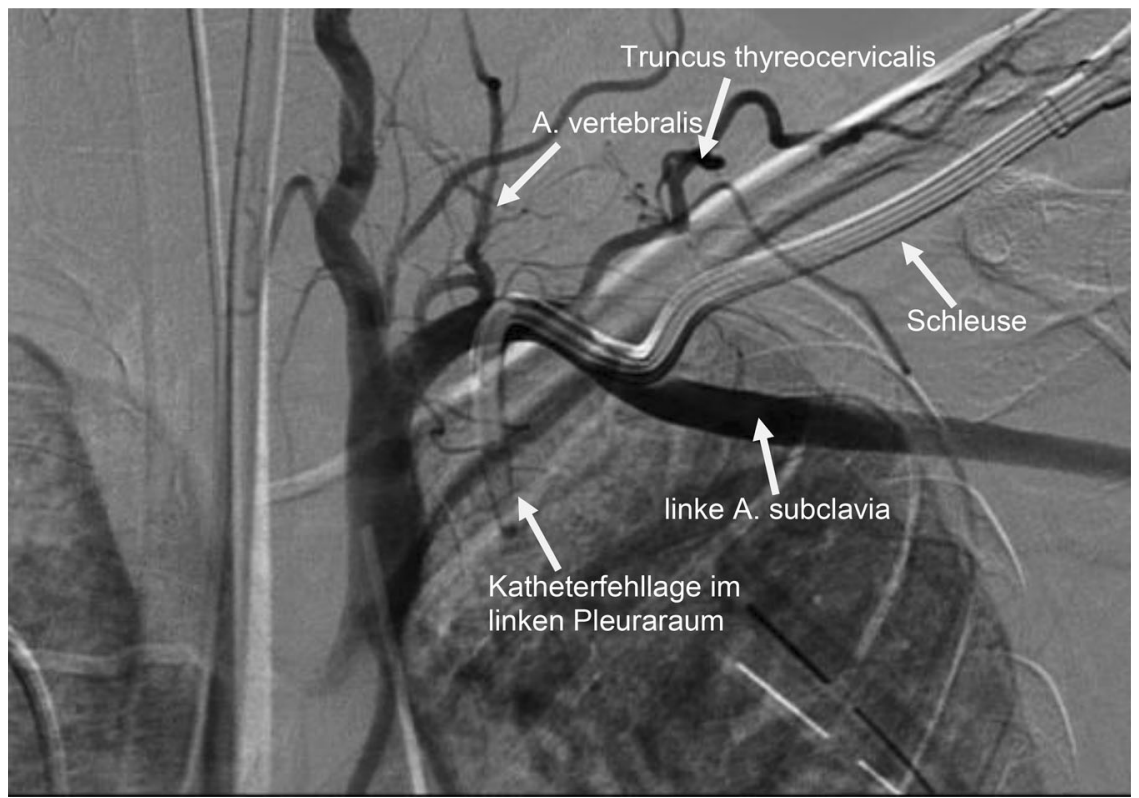

Abb. 2 × Fall 1; Fehllage der Katheterschleuse im linken Pleuraraum nach transarterieller Perforation

intrakraniellen Hypertension mit Übergang in den Hirntod in den folgenden $72 \mathrm{~h}$.

\section{Fall 3}

Eine 62-jährige Patientin mit einer dekompensierten Leberzirrhose entwickelte eine Pneumonie. Bei der Anlage eines ZVK in die linke V. subclavia kam es $\mathrm{zu}$ einem Hämatothorax mit einem hämorrhagischen Schock (• Abb. 4). Die Blutung konnte angiografisch zunächst nicht gestillt werden; in einer darauffolgenden CT-Untersuchung erlitt die Patientin einen Kreislaufstillstand und musste kurzzeitig kardiopulmonal reanimiert werden. Anschließend wurden drei Segmente der Pulmonalarterie des linken Oberlappens embolisiert und die Patientin wurde intensivmedizinisch betreut. Es waren weiterhin kontinuierlich Blutprodukte, hoch dosiertes Noradrenalin und Vasopressin zur Kreislaufunterstützung notwendig; in den nächsten Tagen entwickelte die Patientin ein irreversibles Multiorganversagen und verstarb.

\section{Fall 4}

Bei der Anlage eines ZVK in die V. jugularis bei einer 18-jährigen Patientin wurde die rechte A. subclavia perforiert. Es kam zu einem transfusionspflichtigen Hämo- globinabfall $(5,4 \mathrm{~g} / \mathrm{dl})$ und einer intrathorakalen Blutung (• Abb.5); der Kreislauf musste kurzzeitig mit Adrenalin stabilisiert werden. Während der NotfallThorakotomie entwickelte die Patientin eine weite und lichtstarre Pupille links. Nach einer akuten Entlastungsdrainage waren die Pupillen wieder isokor. Aus dem rechten Thorax wurden zwei Liter blutiger Erguss evakuiert. Eine spritzende arterielle Blutung aus der proximalen rechten A. subclavia wurde anschließend übernäht. Die elektive OP wurde später durchgeführt.

\section{Fall 5}

Einer 76-jährigen Patientin wurde für die Durchführung eines ALIF (anterior lumbar interbody fusion) bei Spondylodiscitis ein $\mathrm{ZVK}$ in die rechte V. subclavia gelegt. Während der Operation wurde eine Katheterfehllage im Truncus brachiocephalicus festgestellt. Der Versuch einer angiographischen Katheterbergung blieb erfolglos und es musste eine $\mathrm{He}$ misternotomie durchgeführt werden; der ZVK konnte dabei entfernt werden. Im Aufwachraum erlitt die Patientin einen Kreislaufstillstand, dessen Ursache auch bildgebend nicht geklärt werden konnte, und musste für ca. zwei Minuten kardiopulmonal reanimiert werden. Am zweiten postoperativen Tag war die Patien- tin katecholaminfrei und am dritten Tag wurde sie problemlos extubiert und auf eine Observationsstation verlegt.

\section{Fall 6}

Einem 45-jährigen Patienten wurde nach einer bihemisphäriellen Subarachnoidalblutung aus einem A. vertebralis Aneurysma ein Flow-Deverter komplikationslos endovaskulär angelegt. Am vierten postoperativen Tag kam es zu einem septischen Schub mit akutem Nierenversagen. Es folgte ein frustraner Anlageversuch eines Quinton-Katheters in die V. subclavia dextra; dabei kam es zu einer starken Blutung aus der V. subclavia mit hämorrhagischem Schock. Der Patient wurde medikamentös reanimiert, massivtransfundiert und anschließend thorakotomiert. Eine $8 \mathrm{~cm}$ lange Gefäßläsion der V. subclavia wurde übernäht und ein großes intrathorakales Hämatom konnte ausgeräumt werden. Der Patient erholte sich komplett.

\section{Diskussion}

Die V. subclavia und die V. jugularis gehören neben der V. femoralis zu den drei gebräuchlichsten zentralvenösen Zugangswegen in der Anästhesiologie und in der Intensivmedizin [2]. Dabei ist eine Vielzahl von relevanten Komplikationen wie z. B. eine Venenthrombose, Pulmonalembolie, System- oder Lokalinfektion, Parästhesien, Hämatothorax, Luftembolie und Pneumothorax möglich, die unter Umständen binnen kurzer Zeit lebensgefährlich sein können. In Einzelfällen entstanden nach ZVKAnlagen in die V. jugularis Komplikationen wie eine "cannot intubate, cannot ventilate" Situation durch ein massives zervikales Hämatom [3], bilateraler Chylothorax [4], Dissektion der A. vertebralis und arteriovenöse Fistelbildung [5]. Bei ZVK-Anlagen in die V. subclavia entstanden in Einzelfällen Komplikationen wie eine Punktion des Aortenbogens mit hämorrhagischem Schock [6], Herzbeuteltamponade [7], Pseudoaneurysma und arteriovenöse Fistelbildung [8].

Die korrekte Platzierung eines zentralvenösen Katheters stellt bei traumatologischen Notfällen eine besondere 
Herausforderung dar, weil erschwerende Faktoren wie großer Zeitdruck durch einen instabilen Blutdruck, Immobilisierung des Patienten und andere ungünstige Rahmenbedingungen dazukommen können. Eine retrospektive Studie an 465 Patienten [9] hat ergeben, dass es bei schwer verletzten Patienten häufiger zu V. subclavia Katheter assoziierten Pneumothoraces und seltener zu V. femoralis Katheter assoziierter Sepsis kam als bei kreislaufstabilen Patienten. Ärzte aus einem Traumazentrum in Korea empfehlen daher, einen V. femoralis Katheter am traumatologischen Patienten so bald als möglich $\mathrm{zu}$ entfernen und gegebenenfalls durch einen V. subclavia Katheter zu ersetzen, um das Risiko für katheterassoziierte Infektionen $\mathrm{zu}$ vermindern [10].

Grundsätzlich sollte die Wahl des Punktionsortes individuell auf den Patienten und den Verwendungszweck des ZVK abgestimmt werden. Dabei sind Kriterien wie die anatomische Zugänglichkeit (z. B. Adipositas, Voroperationen, anatomische Besonderheiten am Hals und Thorax), Volumenstatus des Patienten, individuelle Risikofaktoren (z. B. Blutgerinnung, lokale Infektionen, sowie die geplante Verweildauer des ZVK zu berücksichtigen. Jeder dieser drei Zugangswege ist mit verschiedenen Komplikationen assoziiert. Sowohl die Punktion der V. jugularis als auch die Punktion der V. femoralis ist mit einem höheren Risiko für katheterassoziierte Infektionen [2, 11] und symptomatische Venenthrombosen $[2,12]$ verbunden als ein zentralvenöser Zugang über die V. subclavia. Leitlinien zur Prävention von katheterassoziierten, intravaskulären Infektionen empfehlen bei Erwachsenen einen zentralvenösen Zugang über die V. subclavia [13], der jedoch mit einem höheren Risiko für mechanische Komplikationen assoziiert ist [2]. Während in mehreren randomisierten, kontrollierten Studien [14, 15] bestätigt wurde, dass eine ultraschallgesteuerte Punktion der V. jugularis interna der Landmarkentechnik hinsichtlich des Erfolgs nach der ersten Punktion, Gesamterfolgsrate, Komplikationen, Zeitaufwand und Kosten deutlich überlegen ist, gibt es bezüglich eines möglichen Vorteils einer

Anaesthesist 2016 -65:866-871 DOI 10.1007/s00101-016-0226-8

๑ Der/die Autor(en) 2016. Dieser Artikel ist eine Open-Access-Publikation.

\section{J. Abram · J. Klocker · N. Innerhofer-Pompernigg · M. Mittermayr · M. C. Freund . N. Gravenstein · V. Wenzel \\ Verletzungen herznaher Gefäße durch zentralvenöse Katheter}

\section{Zusammenfassung}

Bei Verletzungen herznaher Gefäße ist ein lebensgefährlicher Verlauf binnen kurzer Zeit möglich. Hierzu zählen u. a. arterielle Gefäßverletzungen bei zentralen Venenpunktionen, die zu einem hämorrhagischen Schock führen können. Wir berichten von sechs Patienten, bei denen Verletzungen der A. und V. subclavia zu lebensgefährlichen Komplikationen führten; ZVKs sind mit einer Vielzahl von Risiken wie z. B. einer Venenthrombose, Pulmonalluftembolie, System- oder Lokalinfektionen, Parästhesien, Hämatothorax, Pneumothorax und cervikalen Hämatomen assoziiert, die nicht immer sofort feststellbar sind. Der Subclaviakatheter geht mit einem etwas kleineren Risiko für katheterassoziierte Sepsis und symptomatische Venenthrombosen einher als Zugänge über die V. jugularis interna und V. femoralis. Allerdings hat der Zugang über die V. subclavia ein relevantes Pneumo- und Hämatothoraxrisiko.
Eine Verletzung der V. oder A. subclavia kann auch bei gewissenhafter und regelrechter Punktion passieren und in lebensgefährlichen Komplikationen resultieren. Daher ist eine sorgfältige Abwägung des Zugangswegs in Relation zum Patienten und zur klinischen Situation erforderlich, um die Inzidenz einer Komplikation so niedrig wie möglich zu halten. Für die Katheterisierung der V. subclavia ist möglicherweise die Punktion der V. axillaris in der Regio infraclavicularis eine gute Alternative, weil eine Ultraschalldarstellung des Zielgefäßes einfacher ist als bei der V. subclavia und die Punktion in viel größerem Abstand von der Lunge durchgeführt werden kann.

\section{Schlüsselwörter}

Zentralvenöse Katheterisierung · V. subclavia · A. subclavia $\cdot$ V. axillaris $\cdot$ Hämatothorax

\section{Injuries to blood vessels near the heart caused by central venous catheters}

\section{Abstract}

Injuries to blood vessels near the heart can quickly become life-threatening and include arterial injuries during central venous puncture, which can lead to hemorrhagic shock. We report 6 patients in whom injury to the subclavian artery and vein led to lifethreatening complications. Central venous catheters are associated with a multitude of risks, such as venous thrombosis, air embolism, systemic or local infections, paresthesia, hemothorax, pneumothorax, and cervical hematoma, which are not always immediately discernible. The subclavian catheter is at a somewhat lower risk of catheter-associated sepsis and symptomatic venous thrombosis than approaches via the internal jugular and femoral veins. Indeed, access via the subclavian vein carries a substantial risk of pneumo- and hemothorax. Damage to the subclavian vein or artery can also occur during deliberate and inadvertent punctures and result in life-threatening complications. Therefore, careful consideration of the access route is required in relation to the patient and the clinical situation, to keep the incidence of complications as low as possible. For catheterization of the subclavian vein, puncture of the axillary vein in the infraclavicular fossa is a good alternative, because ultrasound imaging of the target vessel is easier than in the subclavian vein and the puncture can be performed much further from the lung.

\section{Keywords}

Central venous catheterization - Subclavian vein . Subclavian artery . Axillary vein . Hemothorax ultraschallgesteuerten Punktion der V. subclavia gegenüber der Landmarkentechnik bisher keine eindeutigen Beweise. Zwei prospektive, randomisierte Studien $[16,17]$ haben eine Überlegenheit der ultraschallgestützten Punktion der V. subclavia gegenüber der Land- markentechnik ergeben. Experten der American Society of Anesthesiologists sind sich jedoch über den Vorteil einer ultraschallgestützten Punktion der V. subclavia nicht einig und geben in ihren Leitlinien keine klare Empfehlung ab [18]. Das Cochrane-Netzwerk [19] 

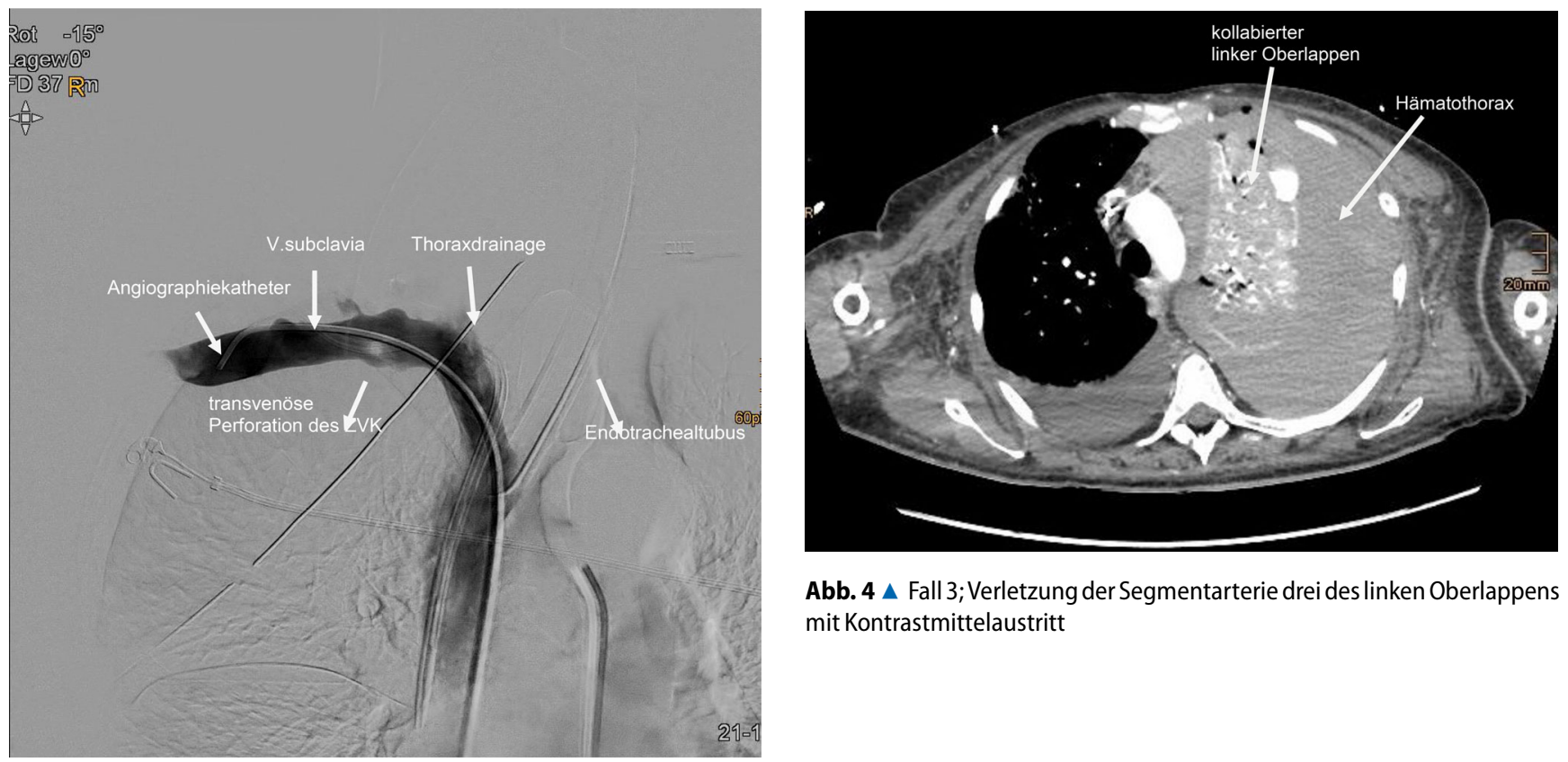

Abb. $4 \Delta$ Fall 3; Verletzung der Segmentarterie drei des linken Oberlappens mit Kontrastmittelaustritt

Abb. 3 A Fall 2; transvaskuläre Perforation des ZVK durch die V. subclavia
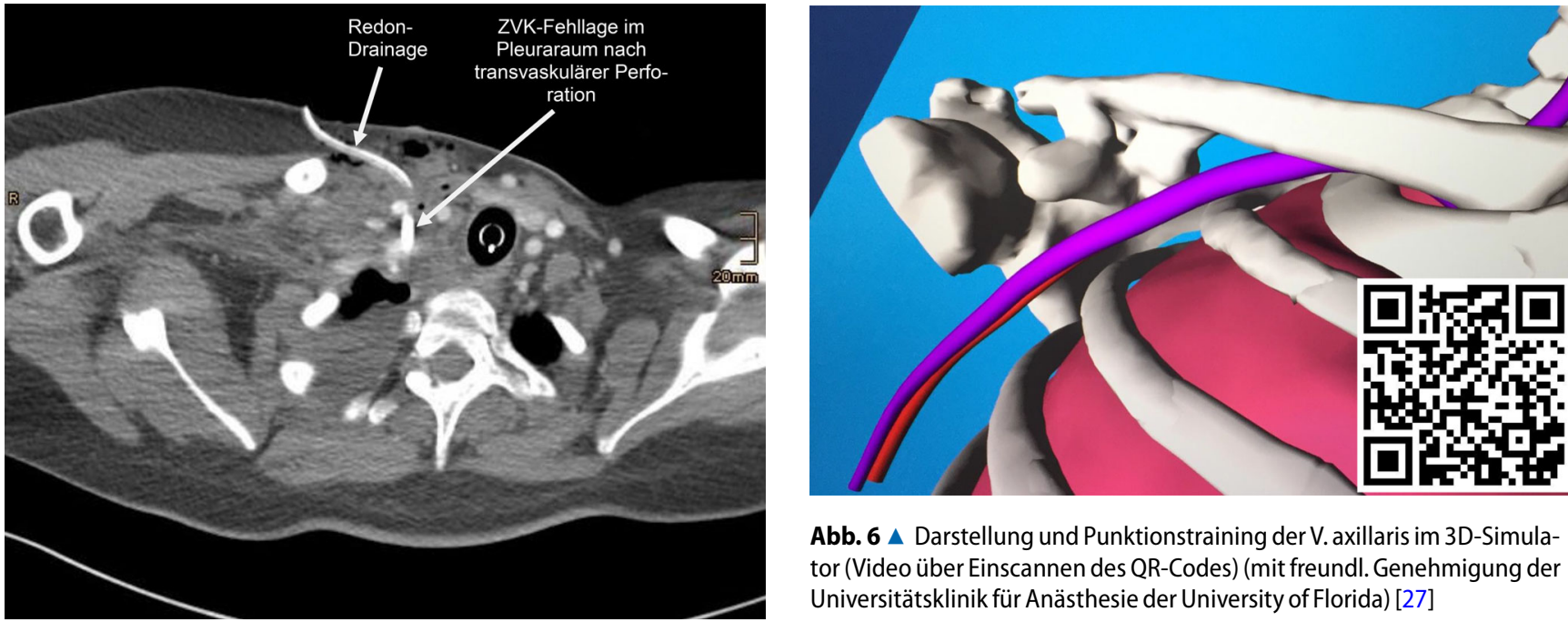

Abb. $6 \Delta$ Darstellung und Punktionstraining der V. axillaris im 3D-Simulator (Video über Einscannen des QR-Codes) (mit freundl. Genehmigung der Universitätsklinik für Anästhesie der University of Florida) [27]

Abb. $5 \Delta$ Fall 4; ZVK-Fehllage nach transvaskulärer Perforation

kam 2015 allerdings zu dem Schluss, dass zweidimensionaler Ultraschall als Hilfe zur Punktion der V. subclavia gegenüber der Landmarkentechnik ein niedrigeres Risiko für eine arterielle Punktion und Hämatombildung aufweist. Gleichzeitig räumten die Autoren dieser CochraneStudie aber auch ein, dass Daten über die Positionierung des ZVK in die V. subclavia durch erfahrene bzw. unerfahrene Ärzte und Studien an Patienten mit hohem Komplikationsrisiko fehlen [19].

Die Sicherheit des V. subclavia Zugangs kann möglicherweise erhöht wer- den, wenn die Punktionsstelle für den ZVK von der V. subclavia in Richtung V. axillaris im Schulterbereich verschoben wird. Sie gilt als Fortsetzung der V. brachialis und geht am lateralen Rand der ersten Rippe in die V. subclavia über (• Abb.6). Die V. axillaris ist im Ultraschall auch longitudinal darstellbar und eine Punktion kann somit unter kontinuierlicher Sicht der Nadelspitze erfolgen (• Abb. 7). Bereits 1987 bezeichnete ein englischer Anästhesist [20] die V. axillaris als möglichen Zugangsweg mit geringem Pneumothoraxrisiko (• Tab. 1). Als Risi- ko dieser Methode nannte er die mögliche akzidentelle Punktion der A. axillaris. Sechzehn Jahre später beschrieben wiederum englische Anästhesisten [21] die Darstellung der V. axillaris im Ultraschall und argumentierten, dass sie einen größeren Abstand von der A. axillaris hat, weiter von der Lunge entfernt ist und daher mehr Sicherheit für eine ZVK-Anlage bietet. Die gleiche Arbeitsgruppe [22] berichtete später von 2586 konsekutive Patienten, denen ein V. axillaris Katheter ultraschallgesteuert gelegt wurde; in $0,41 \%$ der Fälle kam es zu einer arte- 


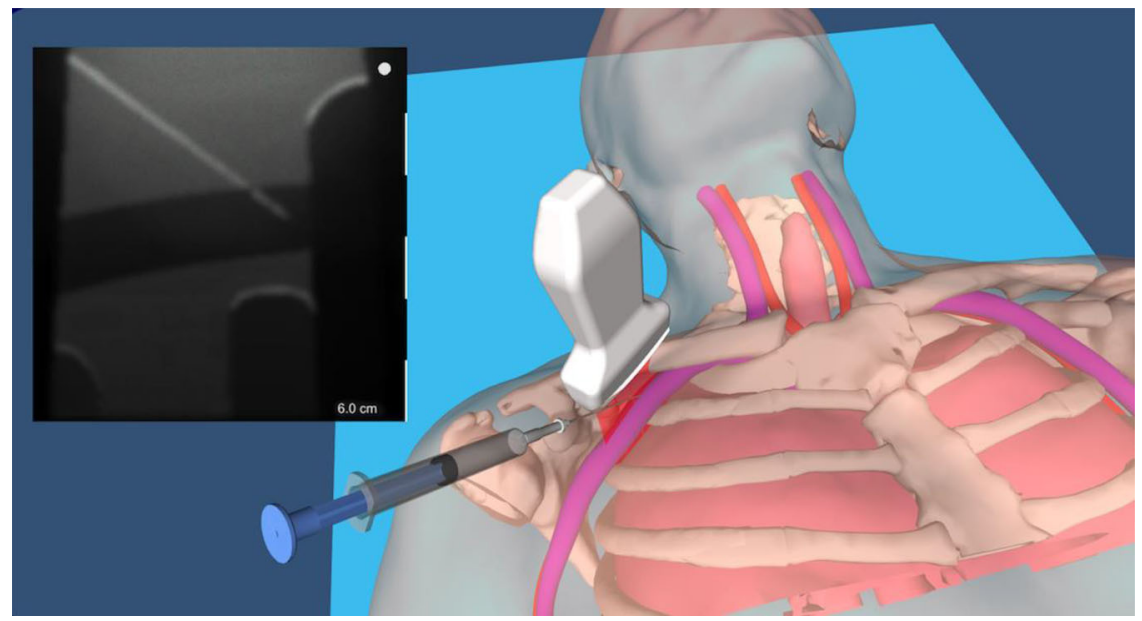

Abb. 7 ॥ Punktion der V. axillaris unter kontinuierlicher Sicht der Nadelspitze (mit freundl. Genehmigung der Universitätsklinik für Anästhesie der University of Florida)

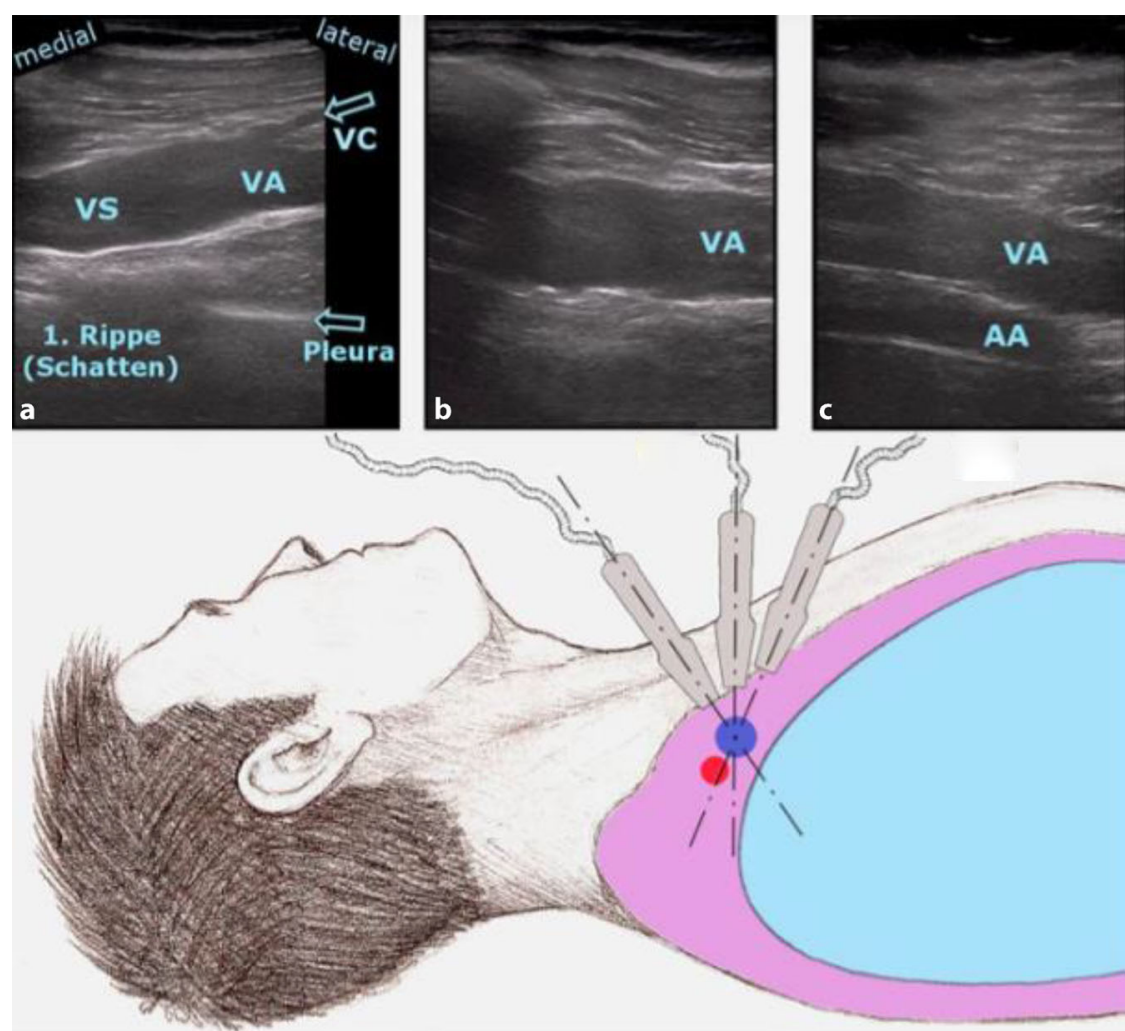

Abb. 8 \& Infraklavikuläre Sonographie des Übergangsbereichs der rechtsseitigen V. axillaris in die V. subclavia. Lange Gefäßachse, Darstellung von drei möglichen Einstellungen: $A$ von kranioventral, $B$ von ventral, $C$ von kaudoventral. $A A$ A. axillaris, VA V. axillaris, VCV. cephalica, VS V. subclavia (mit freundl. Genehmigung der Autoren [24] und des Springer Verlags)

riellen Punktion, die Pneumothoraxrate betrug $0,1 \%$. Dieser Zugangsweg erwies sich auch bei 105 beatmeten Patienten auf einer ICU als gut machbar [23]. Gaus et al. [24] empfehlen dabei die "in plane“ Punktion (• Abb. 8), damit die A. und V. axillaris, die 1. Rippe und die Pleura gut
Die zentralvenöse Katheterisierung ist ein Routineverfahren, das auch bei gewissenhafter und regelrechter Punktion in lebensgefährlichen Komplikationen resultieren kann. In jedem Einzelfall muss die Indikation zum Anlegen eines zentralen Venenkatheters (z. B. Infusion von großen Volumina oder Katecholaminen, Langzeit-Infusionstherapie potenziell gefäßtoxischer Substanzen, hämodynamische Überwachung, Hämofiltration und Dialyse) genau hinterfragt werden. Risiko und Nutzen sollen gegeneinander abgewägt werden und eine kritische Lagekontrolle muss zum Erkennen möglicher Komplikationen durchgeführt werden. Dazu gehören u.a. eine ZVK-Lagekontrolle bei liegendem Führungsdraht mittels Ultraschall, Aspiration nach Entfernung des Führungsdrahtes, Aspiration und Spülen aller Lumina und eine ThoraxRöntgenaufnahme in Exspiration. Indikationsstellung, Patientenaufklärungen, eventuelle Fehlpunktionen, Ultraschallbilder und Nachkontrollen sollen dokumentiert werden. Experten der American Society of Anesthesiologists empfehlen den Einsatz von Checklisten bei zentralvenösen Katheterisierungen [18].

Die Indikation für den zentralen Venenkatheter war bei allen von uns beschriebenen Fällen gegeben (Messung des zentralen Venendruckes und Infusion von großen Volumina bzw. Katecholaminen). Drei von sechs iatrogen induzierten Komplikationen einer ZVKAnlage unserer Fälle erfolgten durch einen Nicht-Anästhesisten. Dabei soll hier keinesfalls die „beste Disziplin“ für eine ZVK-Anlage diskutiert werden, zumal wir aus den uns verfügbaren Zahlen keine belastbare Inzidenz berechnen können und diese Komplikationen auch sehr erfahrenen Anästhesisten passieren können. Insgesamt ist ggf. anzustreben, nur noch eine ZVK-Anlage in mit Ultraschall gut darstellbare Gefäße wie die V. jugularis, V. axillaris und V. femoralis vorzunehmen - unabhängig von ihrer anatomischen Lage. Dabei ist aber auch zu bedenken, dass wie in unserem Fall 4 bei einer ZVK-Anlage über die V. jugularis die V. subclavia verletzt werden kann. 
Tab. 1 Ergebnisse aus bisherigen Studien über axilläre Zugänge

\begin{tabular}{|llllll}
\hline Autor & Anzahl & Erfolgsrate & Pneumothorax & $\begin{array}{l}\text { Arterielle } \\
\text { Punktion }\end{array}$ & Neuralgie \\
\hline Nickalls et al. [20] & 14 & $92,8 \%$ & 0 & 0 & k. A. \\
\hline Taylor et al. [25] & 102 & $96,1 \%$ & 0 & $4,9 \%$ & $2 \%$ \\
\hline Sharma et al. [26] & 200 & $97 \%$ & 0 & $1,5 \%$ & $1 \%$ \\
\hline O'Leary et al. [22] & 1923 & k. A. & $0,1 \%$ & $0,41 \%$ & k. A. \\
\hline Glen et al. [21] & 125 & $94 \%$ & 0 & $1 \%$ & k. A. \\
\hline
\end{tabular}

k. A. keine Angaben

\section{Fazit für die Praxis}

Eine Verletzung der V. und A. subclavia kann auch bei gewissenhafter und regelrechter Punktion passieren und in lebensgefährlichen Komplikationen resultieren. Daher ist eine sorgfältige Abwägung des Zugangswegs eines ZVK in Relation zum Patienten und zur klinischen Situation erforderlich, um die Inzidenz einer ZVK-Komplikation so niedrig wie möglich zu halten. Für die Katheterisierung der V. subclavia könnte eine Punktion der V. axillaris in der Regio infraclavicularis eine Alternative sein, weil eine Ultraschalldarstellung des Zielgefäßes einfacher ist als bei der V. subclavia und die Punktion in größerem Abstand von der Lunge durchgeführt werden kann.

\section{Korrespondenzadresse}

\section{Prof. Dr. V. Wenzel, M. Sc., FERC}

Bodensee Medizin Campus, Klinik für Anästhesie, Intensivmedizin, Notfallmedizin und Schmerztherapie Röntgenstraße 2, 88048 Friedrichshafen, Deutschland v.wenzel@klinikum-fn.de

Open access funding provided by University of Innsbruck and Medical University of Innsbruck.

\section{Einhaltung ethischer Richtlinien}

Interessenkonflikt. J. Abram, J. Klocker, N. Innerhofer-Pompernigg, M. Mittermayr, M. C. Freund, N. Gravenstein und V. Wenzel geben an, dass kein Interessenkonflikt besteht.

Dieser Beitrag beinhaltet keine von den Autoren durchgeführten Studien an Menschen oder Tieren.

Open Access. Dieser Artikel wird unter der Creative Commons Namensnennung 4.0 International Lizenz (http://creativecommons.org/licenses/by/4.0/deed. de) veröffentlicht, welche die Nutzung, Vervielfäl- tigung, Bearbeitung, Verbreitung und Wiedergabe in jeglichem Medium und Format erlaubt, sofern Sie den/die ursprünglichen Autor(en) und die Quelle ordnungsgemäßnennen, einen Linkzur Creative Commons Lizenz beifügen und angeben, ob Änderungen vorgenommen wurden.

\section{Literatur}

1. Pernter P, Gostner P, Egarter Vigl E, Rühli FJ (2007) Radiologic proof for the Iceman's cause of death (ca. 5'300 BP). J Archaeol Sci 34:1784-1786

2. Parienti JJ, Mongardon N, Mégarbane B, Mira JP Kalfon $P$, Gros A, Marqué $S$, Thuong M, Pottier V, Ramakers M, Savary B, Seguin A, Valette $X$, Terzi N, Sauneuf B, Cattoir V, Mermel LA, du Cheyron D, 3SITES Study Group (2015) Intravascular complications of central venous catheterization by insertion site. NEngl J Med 373(13):1220-1229

3. Engelen C, Trebes C, Czarnecki S, Junger A (2016) Bleeding during central venous catheterization: Cannot intubate, cannot ventilate due to massive cervical hematoma. Anaesthesist 65(3):198-202

4. Saxena P, Shankar S, Kumar V, Naithani N (2015) Bilateral chylothorax as a complication of internal jugular vein cannulation. Lung India 32(4):370-374

5. Finlay DJ, Sanchez LA, Sicard GA (2002) Subclavian artery injury, vertebral artery dissection, and arteriovenous fistulae following attempt at central line placement. Ann Vasc Surg 16(6):774-778

6. Kastler A, Chabanne R, Azarnoush K, Cosserant B, Camilleri L, Boyer L, Chabrot P (2012) Arterial injury complicating subclavian central venous catheter insertion. J Cardiothorac Vasc Anesth 26(1):101-103

7. Maschke SP, Rogove HJ (1984) Cardiac tamponade associated with a multilumen central venous catheter. Crit Care Med 12(7):611-613

8. Guilbert MC, Elkouri S, Bracco D, Corriveau MM, Arterial trauma during central venous catheter insertion: Case series, review and proposed algorithm. JVasc Surg 48(4):918-925

9. Choron RL, Wang A, Van Orden K, Capano-Wehrle L, Seamon MJ (2015) Emergency central venous catheterization during trauma resuscitation: $A$ safety analysis by site. Am Surg 81(5):527-531

10. Youn SH, Lee JC, Kim Y, Moon J, Choi Y, Jung K (2015) Central venous catheter-related infection in severe trauma patients. World J Surg 39(10):2400-2406

11. McKinley S, Mackenzie A, Finfer S, Ward R, Penfold J (1999) Incidence and predictors of central venous catheter related infection in intensive care patients. Anaesth Intensive Care 27(2):164-169

12. Timsit JF, Farkas JC, Boyer JM, Martin JB, Misset B, Renaud B, Carlet J (1998) Central vein catheterrelated thrombosis in intensive care patients: Beaudoin N, Dubois MJ, Bruneau L, Blair JF (2008)
Incidence, risks factors, and relationship with catheter-related sepsis. Chest 114(1):207-213

13. O'Grady NP, Alexander M, Burns LA, Dellinger EP, Garland J, Heard SO, Lipsett PA, Masur H, Mermel LA, Pearson ML, Raad II, Randolph AG, Rupp ME, Saint S, Healthcare Infection Control Practices Advisory Committee (2011) Guidelines for the prevention of intravascular catheter-related infections. Am J Infect Control 39(4 Suppl 1):S1-34

14. Hind D, Calvert N, McWilliams R, Davidson A, Paisley S, Beverley C, Thomas S (2003) Ultrasonic locating devices for central venous cannulation: Meta-analysis. BMJ327(7411):361

15. Karakitsos D, Labropoulos N, De Groot E, Patrianakos AP, Kouraklis G, Poularas J, Samonis G, Tsoutsos DA, Konstadoulakis MM, Karabinis A (2006) Realtime ultrasound-guided catheterisation of the internal jugular vein: A prospective comparison with the landmark technique in critical care patients. Crit Care 10(6):R162

16. Fragou M, Gravvanis A, Dimitriou V, Papalois A Kouraklis G, Karabinis A, Saranteas T, Poularas J, Papanikolaou J, Davlouros P, Labropoulos N, Karakitsos D (2011) Real-time ultrasoundguided subclavian vein cannulation versus the landmarkmethod in critical care patients: A prospective randomized study. Crit Care Med 39(7):1607-1612

17. Gualtieri E, Deppe SA, Sipperly ME, Thompson DR (1995) Subclavian venous catheterization: Greater success rate for less experienced operators using ultrasound guidance. Crit Care Med 23(4):692-697

18. Rupp SM, Apfelbaum JL, Blitt C, Caplan RA, Connis RT, Domino KB, Fleisher LA, Grant S, Mark JB, Morray JP, Nickinovich DG, Tung A, American Society of Anesthesiologists Task Force on Central Venous Access (2012) Practice guidelines for central venous access: a report by the American Society of Anesthesiologists Task Force on Central Venous Access. Anesthesiology 116(3):539-573

19. Brass P, Hellmich M, Kolodziej L, Schick G, Smith AF (2015) Ultrasound guidance versus anatomical landmarks for subclavian or femoral vein catheterization. Cochrane Database Syst Rev 1:CD011447. doi:10.1002/14651858

20. Nickalls RW (1987) A new percutaneous infraclavicular approach to the axillary vein. Anaesthesia 42(2):151-154

21. Galloway S, Bodenham A (2003) Ultrasound imaging of the axillary vein - anatomical basis for central venous access. Br J Anaesth 90(5):589-595

22. O'Leary R, Ahmed SM, McLure H, Oram J, Mallick A, Bhambra B, Bodenham AR (2012) Ultrasoundguided infraclavicular axillary vein cannulation: $A$ useful alternative to the internal jugular vein. $\mathrm{Br} \mathrm{J}$ Anaesth 109(5):762-768

23. Glen H, Lang I, Christie L (2015) Infraclavicular axillary vein cannulation using ultrasound in a mechanically ventilated general intensive care population. Anaesth Intensive Care 43(5):635-640

24. Gaus P, Heß B, Müller-Breitenlohner H (2015) Ultrasound-guided infraclavicular venipuncture at the junction of the axillary and subclavian veins. Anaesthesist 64(2):145-151

25. Taylor BL, Yellowlees I (1990) Central venous cannulation using the infraclavicular axillary vein. Anesthesiology 72(1):55-58

26. Sharma A, Bodenham AR, Mallick A (2004) Ultrasound-guided infraclavicular axillary vein cannulation for central venous access. Br J Anaesth 93(2):188-192

27. Lampotang S, Lizdas DE, Gravenstein N (2011) Video of mixed simulation for placement of CVL needle. (University of Florida CSSALT) https:// www.youtube.com/watch?v=0ITIFbiiwRs 